\title{
Hand-Mixing with Chopsticks: Regulations of Hand Movements by Ingredient Properties and Tool Length
}

\author{
${\text { Kiyohide } \text { Ito }^{*} \text { Hiroyuki Mishima }}^{\dagger}$ \\ (*)Future University Hakodate, JAPAN \\ (†)Waseda University, JAPAN \\ E-mail:itokiyo@fun.ac.jp,mishima@waseda.jp
}

\begin{abstract}
This study was designed to demonstrate the process of mixing different kinds of ingredients, particularly focusing on hand movements that manage chopsticks. Participants were requested to stir two combinations of ingredients: oil and water, as well as brown and white sugar by using either short or long chopsticks. Motion data were analyzed by three methods: the spectral analysis (short-term Fourier transform: $S T F T)$, the recurrence quantification analysis (RQA), and the detrended fluctuation analysis (DFA). It was found that hand motions in mixing varied flexibly according to the relationship between the length of tool (short/long) and the properties of stirred ingredients.
\end{abstract}

\section{Introduction}

Scientific studies on 'mixing' have been made chiefly in chemical engineering field. A major purpose of those studies is to develop effective mixing technique, by which substances with different properties are efficiently mixed to combine. There are various studies in chemical engineering on shapes of mixing vessels and impellers [1], as well as power consumption while mixing [2].

Meanwhile, little research has been carried out on mixing skills in our daily life. Taking into account that we often mix ingredients to make sauce and cocktails, melt sugar into coffee as well as flour into water, it is hypothesized that the skill of hand movement plays an important role in mixing. Nosaka et al. [3] studied correlations between the quality of finished white sauce and hand movements while cooking, by comparing the stirring process of French chefs to that of non-chefs. They measured the stirring velocity and duration time. It was found that finishing quality of white sauce was strongly correlated with stirring velocity: chefs stirred their tools at high velocity to cook quality sauce.
Some studies in chemical engineering point out that mixing in vessels requires linear and discontinuous motion for uniform mixing. It is considered that the findings can be suitable for a person's action to mix. In other words, the action as the chaotically or dynamically structured mixing process will play an important role in uniform mixture. Besides, the way of using a tool should be also crucial. We typically use tool, for example by using a spoon to mix coffee with sugar or milk, or using a spatula to cook white sauce. Therefore, mixing should be regarded as the combination of hand movements and use of a tool. From this point of view, it is necessary to describe the movements of hand and tool by a kinesiological approach so as to reveal a process which allows for uniform mixture.

This study aimed to identify effects of different ingredients and tool types on the hand movement for uniform mixing. For this purpose, we analyzed hand motions while stirring two ingredients.

\section{Methods}

\subsection{Participants}

Participants were four undergraduate students, three males and one female.

\subsection{Experimental Conditions}

Four conditions were presented: two types of ingredient and two different tool lengths. For ingredients, we used the same amount $(50 \mathrm{ml})$ of water and oil ("the OW condition"), and sugar of two different colours (60 g white, $60 \mathrm{~g}$ brown) ("the SS condition"). We used kitchen chopsticks with different length as mixing tool: long chopsticks $(33 \mathrm{~cm}$, 'the long condition') and short chopsticks $(18 \mathrm{~cm}$, 'the short condition').

This is an Open Access article distributed under the terms of the Creative Commons Attribution-Noncommercial License 3.0, which permits unrestricted use, distribution, and reproduction in any noncommercial medium, provided the original work is properly cited. 


\subsection{Procedure}

Participants were asked to stand in front of a table on which bowls were placed. The height of the tabletop was adjusted to $10 \mathrm{~cm}$ below the participant's elbow height. A bowl $(20 \mathrm{~cm}$ in diameter, $8 \mathrm{~cm}$ in depth) contained ingredients with one of the two conditions (OW or SS); water and oil or two colours of sugar were separated and not yet mixed. The participants were asked to stir a bowl of ingredients in each trial with their right hand holding either long or short chopsticks. They were required to continue the action at least 60 seconds and declare when they recognized the ingredients were uniformly stirred. Each subject made two trials in an individual condition so that they made 8 trials altogether, using either long or short chopsticks to stir either the OW or SS condition per trial.

\subsection{Data collection and Analyses}

Hand movements while stirring were recorded by the electromagnetic motion tracking device (Polhemus, PATRIOT, with the sampling rate of $60 \mathrm{~Hz}$ ), attached on the back of their right hands.

Palmar/dorsal flexion (circular motion angle) of a participant's wrist were analyzed by using three methods: (1) short-term Fourier transform (STFT) analysis was performed to compare short-term power spectrums between four conditions; (2) recurrence quantification analysis (RQA) to detect each dynamic system of stirring motion; and (3) detrended fluctuation analysis (DFA) to investigate strengths in temporal correlation of fluctuations of hand motion among various time-range scales.

\section{Results}

\subsection{Spectrum Analysis (short-term Fourier transform)}

We performed the STFT analysis for $50 \mathrm{~s}$ out of 60 $\mathrm{s}$ in each trial. STFT parameters were specified as follows: window size $=3 \mathrm{~s}$, window shift $=0.4 \mathrm{~s}$, window function $=$ hanning. Representatives of STFT were shown in Figures 1 and 2.

Figures 1 (a) and (b) illustrate the short-term power spectrums of short and long chopsticks in the OW condition. When short chopsticks were used, power was distributed in wide range of frequencies in the beginning stage of stirring, but the peak power shifted to relatively lower frequencies at the last stage, as shown in Figure 1 (a). On the contrary, the width of the strong power distribution on using long chopsticks was narrower than that of short chopsticks. Furthermore, the range of frequencies on which power concentrated remained stable throughout the stirring process, as given in Figure 1 (b).

Figures 2 (a) and (b) represent the short-term power spectrums under two ingredient conditions by using short chopsticks. The result of the OW condition indicated that the peaks of power were limited to the extremely narrow bandwidth of frequencies that was consistent and did not change throughout the stirring process, found in Figure 2 (a). In contrast, the range of frequencies that had strong power was narrow at the beginning of stirring; however, the range became gradually wider toward the end of the process. This qualitative analysis indicates that chopstick lengths and ingredient types synergically affect on hand movements in mixing, probably, they may be interactive.

\subsection{Recurrence Quantification Analysis}

We conducted the recurrence quantification analysis (RQA) to describe recurring patterns in the time series of stirring movement. RQA is a method of non-linear and multi-dimensional time series analysis [4]-[6]. For this analysis, each series of the data was normalized and the following RQA parameters were used: embedding dimension $=5$, delay $=4(0.067 \mathrm{~s})$, radius $=$ $0.75 \mathrm{SD}$, norm $=$ Euclidian, window size $=360$ samples $(6 \mathrm{~s})$, window shifting value $=60$ samples $(1$ s).

Figure 3(a) illustrates mean recurrence rates of moving RQA in each condition. Figure 3(b) illustrates mean determinism. Recurrence rate was higher in the short condition than in the long condition, and higher in the SS condition than in the OW condition. Determinism was higher in the short condition than in the long condition.

As for the recurrence rate, the statistically significant difference between ingredients was found $(F(1,3)=14.56, p<.05)$. The difference between chopstick lengths reached a marginally significant level $(F(1,3)=5.74, p<.10)$. As for determinism, there was a significant difference between chopstick lengths $(F(1,3)=17.42, p<.05)$.

To sum up, it is suggested that short chopsticks make relatively regular movements, and the mixing movement becomes less recurrent in the SS condition, which often accompanies a kinetic transition. 


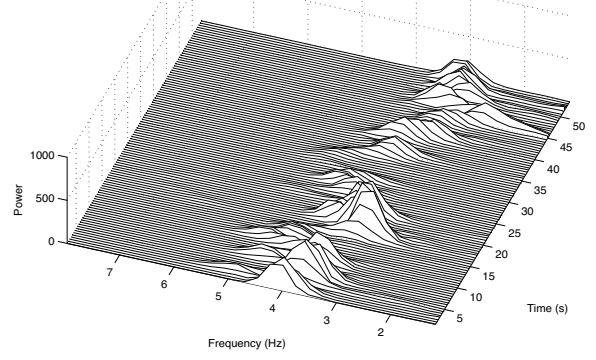

(a) Hand motion by using short chopsticks

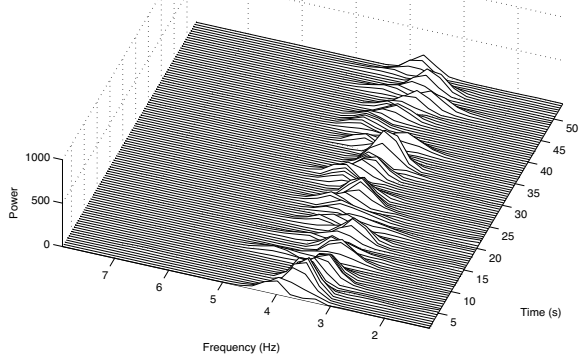

(b) Hand motion by using long chopsticks

Figure 1. Representatives of results of short-term Fourier transform under the OW condition

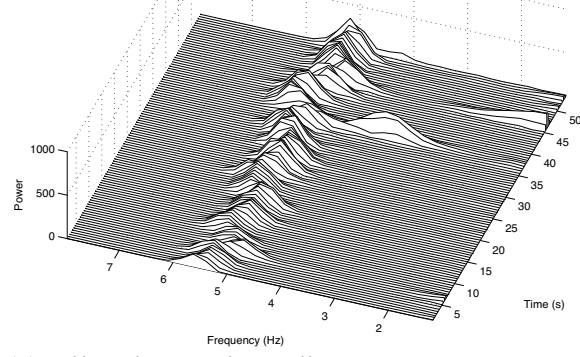

(a) Oil and water ingredients

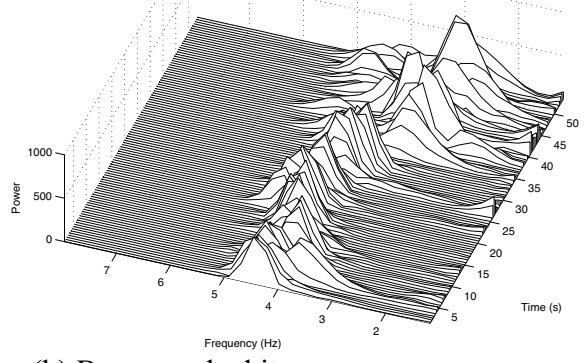

(b) Brown and white sugars

Figure 2. Representatives of results of short-term Fourier transform under the short condition.

\subsection{Detrended Fluctuation Analysis (DFA)}

We performed DFA in order to determine whether or not hand movement patterns maintain a certain stable structure, or long-range correlations, through the whole stirring process. If hand movements are somehow structured, fluctuations of hand movements are auto-correlated in the range of time (short or long) scale. If there are no structures, hand movements fluctuate randomly. DFA has been applied to analyze physiological and behavioral data of human beings (e.g., [7], [8]). We analyzed the series of data for first $20 \mathrm{~s}$ ('the first stage') and for $20 \mathrm{~s}$ from 35 th to 55 th $\mathrm{s}$ ('the second stage') out of $60 \mathrm{~s}$ in each trial. Figure 4 shows examples of DFA, where $x$ refers to the number of data samples, $y$ refers to the fluctuations of the hand movements. Accordingly, dots and circles indicate fluctuation values corresponding to data samples. Dots refer to the result of the short condition, and circles refer to that of the long condition. Regression lines (solid lines in Figure 4) were fitted by the least-squares method. When computing the regression line, the smallest window size was $0.5 \mathrm{~s}$ in order to remove artifacts of high frequency noise. Hence, each window size was varied from $0.5 \mathrm{~s}$ to $4 \mathrm{~s}$. The slope of the regression line $(\alpha)$ was used as a measurement of a statistical analysis. Generally, $\alpha=0.5,1.0$, and 1.5 mean white noise, pink noise, and brown noise, respectively.

We then computed the mean $\alpha$ of the both first and second stages in each of four conditions (Figure 5). Figure 5 indicated that the mean $\alpha$ in the SS condition was 0.8883 for the short condition, while 1.4207 for the long condition. In the OW condition, the mean $\alpha$ was 1.0377 for the short condition, and 1.2507 for the long condition.

The main effect of chopsticks lengths was statistically significant $(F(1,3)=53.87, p<.01)$. There was marginally significant interaction between lengths and ingredients $(F(1,3)=9.20, p<.10)$. However, any significant difference was not found between two stages and ingredient conditions.

As a result of DFA, it was found that different lengths of chopsticks varied the structure of circular motion in mixing. When participants used long chopsticks, correlation was higher in a long time range, leading that hand movement was structured in large scale. Meanwhile, using short chopsticks generated no dominant fluctuation correlation, rather making hand movement random or chaotic.

Detrended fluctuation analysis has demonstrated that chopsticks length affects the mixture of liquid/solid ingredients, and that the mixing strategy depends upon the hand movement and the length of chopsticks used to make the mixture. 


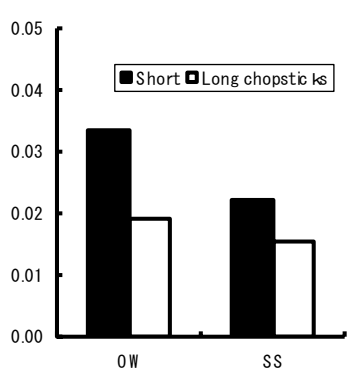

(a) RecurrenceRate

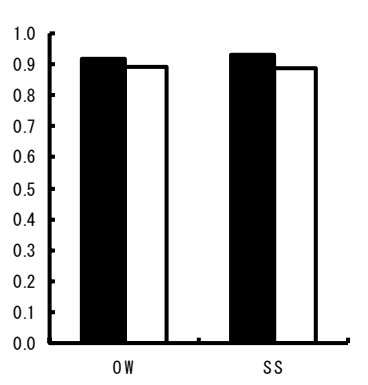

(b) Determinism

Figure 3. Mean Measures under four conditions.

\section{Conclusion}

This study aimed to analyze the motion of uniformly mixing ingredients with different properties (two kinds of liquid/solid materials) and to reveal the effective mixing method.

On the one hand, the mixture of oil and water transforms both colour and viscosity of the original ingredients. This process makes persons perceive the state of mixture both visually (seeing colours change) and haptically (feeling ingredients heavy via chopsticks). On the other hand, a mixture of two colours of sugar varies only colour of the ingredients but viscosity remains the same. This colour change only allows a person to specify the mixture via only visual modality.

In addition to ingredient conditions, we had two different chopstick lengths to find out an appropriate method of using tools to mix different ingredients.

We performed three types of analysis, concluding that: (1) mixing liquids by using short chopsticks provide the most regular motion, stirring with high velocity; and (2) mixing sugars by using long chopsticks provide rather non-structured and irregular motion, stirring by both high and low circular movements in the beginning and then shifting to lower circular movement. In other words, stirring quickly varied from the fast to the slow velocity in the first stage then, slowed to the end.

This study has demonstrated that tools and ingredients are interactive in uniform mixing. This also suggests that the goal-directed motion of mixing can be flexible to accommodate ingredients and tool properties, which corresponds to the flexibility that Gibson [9] pointed out as one of the intelligences animals have in perceptual learning.

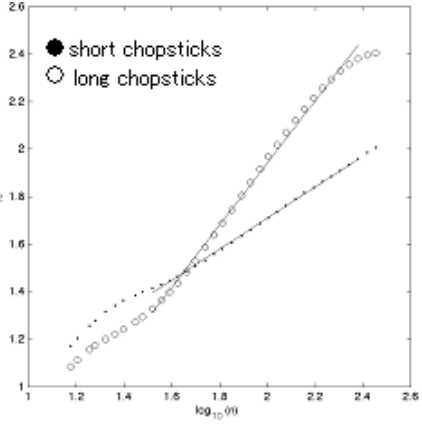

Figure 4. Representatives of results of DFA

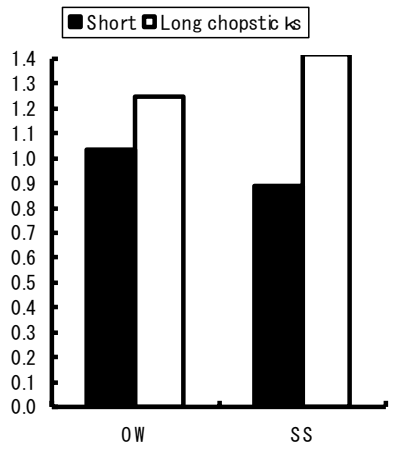

Figure 5. Mean alphas under four conditions.

\section{Acknowledgement}

We are grateful to Saori Ariizumi, Yasuko Nagasaki, Yukie Sumiyama, and Miho Takahashi for precious assistances.

\section{References}

[1] D. Devakumar, D. and K. Saravanan. Study and Design of Impellers for Multiphase Reactors. Mod. Appl. Sci., 2(5), 99-121, 2008.

[2] S. Foucault, et al. Power Characteristics in Coaxial Mixing: Newtonian and Non-Newtonian Fluids. Ind. Eng. Chem. Res., 44(14), 5036-5043, 2005.

[3] C. Nosaka, et al. Effect of Cooking Process on Physical Properties of White Sauce: Comparison between a chef and an unskilled subject for mixing process. J. Cook. Sci. Jpn. 34(1), 10-16, 2001.

[4] N. Marwan and J. Kurths. Nonlinear analysis of bivariate data with cross recurrence plots, Physics Letter. A, 302(5-6), 299-307, 2002.

[5] K. Shockley, et al. Mutual interpersonal postural constraints are involved in cooperative conversation. J. Exp. Psychol Human, 29(2), 326-332. 2003.

[6] J. P. Zbilut and C. L. Webber Jr. Embeddings and delays as derived from quantification of recurrence plots. Physics Lett. A, 171, 199-203, 1992.

[7] C. K., Peng, et al. Quantifying Fractal Dynamics of Human Respiration: Age and Gender Effects. Ann. of Biomed. Eng., 30, 683-692, 2002.

[8] Roerdink, M., et al. (2006). Dynamical Structure of center-pressure trajectories in patients recovering from stroke. Exp. Brain Res. 174, 256-269.

[9] Gibson, E. J. (1997). An Ecological Psychologist's Prolegomena for Perceptual Development: A Functional Approach. In C. Dent-Read \& P. Zukow-Goldring (Eds.), Evolving Explanations of Development: Ecological Approaches to Organism-Environment Systems (pp.2345). Washington, D.C.: APA. 